\section{Anleitung für den Benutzer}

Das „Gesamtverzeichnis des deutschsprachigen Schrifttums (GV) 1700-1910" ist alphabetisch nach Personennamen und anonymen Sachtiteln in grammatischer Wortfolge geordnet. Diese Ordnung entspricht weitgehend den sog. "Preußischen Instruktionen" (= Instruktionen für die alphabetischen Kataloge der preußischen Bibliotheken; im folgenden kurz „PI“ genannt).

Bei einer kleinen Anzahl von Titeln (sicher weniger als $1 \%$ ) müssen jedoch Abweichungen von den PI beachtet werden, weil den in den Quellenbibliographien von Heinsius, Kayser und Hinrichs verzeichneten Titeln beim Entstehen dieser Verzeichnisse andere, nämlich buchhändlerische Ordnungsregeln zugrunde lagen und die Titelaufnahmen aus vielen Alphabeten sukzessive in ein einziges Alphabet geordnet werden.

Die wichtigsten Abweichungen von den $P I$ sind jene, die der Einheitlichkeit wegen bei allen im „GV 1700-1910“ verzeichneten Titeln beachtet werden, unabhängig davon, ob die einzelnen Titel ursprünglich nach buchhändlerischen Regeln oder nach den PI geordnet waren. Diese Abweichungen betreffen:

\section{Das Alphabet}

Umlaute werden wie ihre Grundbuchstaben geordnet, unabhängig davon, ob sie in einem oder in zwei Buchstaben geschrieben sind. Das bedeutet: $\ddot{a}=a ; a e=a ; ~ \ddot{~}=$ $\mathrm{o} ; \mathrm{oe}=\mathrm{o} ; \ddot{\mathrm{u}}=\mathrm{u} ; \mathrm{ue}=\mathrm{u}$.

z. B.: Andersen
Aenderung
Anderungen
Ast
Aesthetik
Wort
Wörter
Wörterbuch
Worterklärungen
Wurzbach
Würzburg
Wurzel
Wust
Wüste

Unterscheiden sich zwei Wörter nur dadurch, daß in dem einen ein Selbstlaut und in dem anderen an derselben Stelle der zugehörige Umlaut steht, so wird das Wort mit dem Selbstlaut vor dem mit dem Umlaut geordnet.

$\begin{array}{ll}\text { z. B.: } & \text { Bruder } \\ \text { Bridder } \\ \text { Not } \\ \text { Note } \\ \text { Nöte } \\ \text { Noten }\end{array}$

[Diese Abweichung gegenüber dem $\S 22$ der PI entspricht den im deutschen Sprachraum außerhalb der Bibliotheken allgemein üblichen Alphabetisierungsregeln, nach denen auch so renommierte Nachschlagewerke wie z. B. Brockhaus, Duden und Meyer geordnet sind. - Im übrigen erfolgt die Ordnung von $B$ als ss und die Behand- lung von $i$ und $j$ als demselben Buchstaben sowohl nach den buchhändlerischen Regeln als auch nach den PI.]

\section{Gleiche Namen verschiedener Verfasser}

Verschiedene Verfasser mit denselben Vor-und Familiennamen werden nicht unterschieden. Ihre Werke werden so geordnet, als ob sie alle von einem Verfasser stammten. [Abweichung gegenuiber $§ 175$ der PI.]

z. B.: Mayer, Karl: Abenteuer in Indien. 1895.

Mayer, Karl: Bunte Blumen. 1780.

Mayer, Karl: Eisenbahnen in Deutschland. 1875.

Mayer, Karl: Der Westfälische Frieden. 1910.

Mayer, Karl: Indien für junge Leute. 1894.

Mayer, Karl: Der Dreißigjährige Krieg. 1909.

Mayer, Karl: Das Netz der preußischen Eisenbahnen. 1870.

3. Die Ordnung der Schriften innerhalb desselben Verfassernamens

Innerhalb desselben Verfassernamens werden die verschiedenen Einzel- und Gruppenschriften alphabetisch nach vorliegenden Sachtiteln geordnet. [Abweichung gegenüber $\S \S 176$ und 221 der PI.]

z. B.: Shakespeare, William:

- Die beiden Edeln aus Verona.

- The two Gentlemen of Verona.

- Das Gesamtwerk.

- Histories and tragedies.

- Die Komödie der Irrungen.

- Komödien.

- Viel Lärm um nichts.

- Die beiden Veroneser.

- Was ihr wollt.

- Das gesammelte Werk.

- Ausgewählte Werke.

- Gesammelte Werke. 1891.

- Gesammelte Werke. 1893.

- Wie es euch gefallt.

- Ein Wintermärchen

- Poetical Works.

- Der Widerspenstigen Zähmung.

Die genannten Abweichungen $1-3$ können bei der Ordnung aller einschlägigen Eintragungen im „GV 17001910 “ strikt befolgt werden, weil sich beim Zusammenordnen der verschiedenen Alphabete dadurch keine grösseren Verschiebungen ergeben. Daß diese Abweichungen immer zu beachten sind, macht die Suche in solchen Fällen problemlos, weil kalkulierbar.

Anders verhält es sich mit Abweichungen, die gleich das erste Ordnungswort (OW) betreffen. Sie gelten nur bei Eintragungen, die im wesentlichen aus den buchhändlerischen Basisbibliographien von Heinsius, Kayser und Hinrichs stammen. Da aus diesen Verzeichnissen die Hauptmasse der Titel stammt, wurde erwogen, PI-Aufnahmen aus anderen Bibliographien nach buchhändlerischen Regeln umzuordnen und damit jeden Titel nur an einer möglichen Stelle im Alphabet unterzubringen. 
Dadurch wäre es allerdings unmöglich geworden, den größten Teil der Quellenbibliographien sukzessive zu bearbeiten, was aus finanziellen und zeitlichen Gründen wichtigste Voraussetzung für das Zustandekommen des „GV 1700-1910“ ist. Bei den folgenden Abweichungen ist also zu beachten, daß sie zwar für die Mehrzahl der einschlägigen Fälle gelten, bei der Minderzahl solcher Fälle aber auch die PI-gemäße Ordnung zugrunde liegen kann.

Dieses Problem wird dadurch entschärft, daß viele einschlägige Titel einfach doppelt verzeichnet werden: ein$\mathrm{mal}$ an der Stelle, an der sie sich in den buchhändlerischen Basisbibliographien finden, zum anderen dort, wo sie in PI-Verzeichnissen eingeordnet sind. Bei der Suche eines Titels, für den eine der folgenden Abweichungen 4-9 in Frage kommt, empfiehlt es sich also, zunächst an der Stelle nachzusehen, die in den Abweichungen festgelegt wird. Findet sich der gesuchte Titel dort nicht, dann ist auch noch an der Stelle nachzuschlagen, die nach den PI zutrifft.

\section{Verschiedene Formen für dasselbe Wort}

Das erste OW einer Sachtitelschrift gilt in der Orthographie der Vorlage. Das bedeutet, daß ein und dasselbe Wort bei verschiedener Schreibung in der Vorlage auch an verschiedenen Stellen im Alphabet eingeordnet wird. Von der nicht berücksichtigten Form wird jedoch in jedem Fall verwiesen. [Abweichung gegenüber $§ 210$ der PI.]

$$
\begin{aligned}
\text { z. B.: Acten s. a. Akten } \\
\text { Acten des Adels ... } \\
\text { Akten s. a. Acten } \\
\text { Akten des Abendlandes ... }
\end{aligned}
$$

[Ab dem zweiten $\mathrm{OW}$ wird nach der heute geltenden Orthographie geordnet, auch wenn in der Vorlage abweichende Formen stehen.]

\section{Verschiedene Titel für dieselbe Schrift}

5.1. Bei Vorliegen verschiedener Titel in verschiedenen Ausgaben derselben Schrift wird das OW jeweils dem Haupttitel der vorliegenden Ausgabe entnommen. [Abweichung gegenüber § 217 der PI.]

z. B.: Gesetz über die Zwangsversteigerung.

$$
\text { 1. OW: Gesetz. }
$$

z. B.: Das Zwangsversteigerungs-Gesetz.

1. OW: Zwangsversteigerungs-Gesetz.

5.2. Bei Übersetzungen wird das OW dem vorliegenden Übersetzungstitel entnommen. [Abweichung gegenüber $\S 220$ der PI.]

z. B.: Märchen aus Tausendundeiner Nacht. 1. OW: Märchen.

z. B.: Die Schönsten Geschichten aus Tausendundeiner Nacht.

1. OW: Geschichten.

\section{Eingeschränkter Verfasserbegriff}

6.1. Briefsammlungen, in deren Titel nur einer der Korrespondenten oder ein Adressat steht, sowie Briefsamm- lungen, in deren Titel außer dem Adressaten mehr als drei Korrespondenten genannt sind, werden im allgemeinen als Sachtitelschrift behandelt. [Abweichung gegenüber $\S 38$ der PI.]

z. B.: Briefe von und an Friedrich von Gentz. Herausgegeben von Friedrich Carl Wittichen.

1. OW: Briefe. - Vw von: Gentz. - Vw von: Wittichen.

z. B.: Briefwechsel des Herzogs Christoph von Wirtemberg. Herausgegeben von Viktor Ernst.

1. OW: Briefwechsel. $-V w$ von: Ernst.

z. B.: Briefe an Fritz von Stein. Herausgegeben und eingeleitet von Ludwig Rohmann.

1. OW: Briefe. - Vw von: Rohmann.

z. B.: Briefe an Johann Heinrich Merck von Goethe, Herder, Wieland und anderen Zeitgenossen. Herausgegeben von Karl Wagner.

1. OW: Briefe. - Vw von: Wagner.

6.2. Briefwechsel, in deren Sachtitel zwei oder drei Korrespondenten genannt sind, ohne daß einer davon hervorgehoben ist, werden im allgemeinen als Sachtitelschrift behandelt. [Abweichung gegenüber $\S 68 \mathrm{der}$ PI.]

z. B.: Briefwechsel zwischen Schiller und Wilhelm von Humboldt. Herausgegeben von Albert Leitzmann. 1. OW: Briefwechsel. - Vw von: Leitzmann.

6.3. Gesetze, Verordnungen und andere amtliche Veröffentlichungen, in deren Titel ein Urheber genannt ist, werden im allgemeinen als Sachtitelschrift behandelt. [Abweichung gegenüber $\S 58$ der PI.]

z. B.: Die Hofordnung Kurfürst Joachims II. von Brandenburg. Neu herausgegeben und erläutert von Martin Hass.

1. OW: Hofordnung. $-\mathrm{Vw}$ von: Hass.

6.4. Werke, deren Verfasser nicht auf der Haupttitelseite genannt sind, werden nach buchhändlerischen Regeln im allgemeinen als Sachtitelschrift behandelt. Sind Verfasser im Innern eines Werkes genannt oder ermittelt, so werden sie in der Titelaufnahme angegeben und es wird von ihnen auf die Eintragung unter dem Sachtitel verwiesen. [Abweichung gegenüber $\S 70 \mathrm{der}$ PI.]

z. B.: Alt-Duisburg in Wort und Bild. (Von P. Rheindorf.) 1. OW: Alt-Duisburg. - Vw von: Rheindorf.

6.5. Werke, die unter einem Pseudonym erschienen sind, das ohne weiteres als solches erkannt wird, werden im allgemeinen als Sachtitelschrift behandelt. Vom Pseudonym wird verwiesen. [Abweichung gegenüber $\S 141 \mathrm{der}$ PI.]

z. B.: Goldminen-Aktien als Kapital-Anlage. Von Africanus.

1. OW: Goldminen-Aktien. - Vw von: Africanus.

\section{Erweiterter Verfasserbegriff}

7.1. Auszüge aus den Werken eines, zweier oder dreier Verfasser werden unter dem Herausgeber eingeordnet. [Abweichung gegenuiber $\S 37$ der PI.] 
z. B.: Friedrich der Einzige. Ein Charakterbild des grossen Königs in seinen Worten. Zusammengestellt von Prof. Dr. A. Kannengiesser.

\section{OW: Kannengiesser.}

7.2. In der Titelfassung hervorgehobene oder ohne Verfasser genannte Hercusgeber von Sammelwerken werden wie Verfasser behandelt. [Abweichung gegenüber $\S \S 62$ und 65 der PI.]

z. B.: Geschichte der Stadt Potsdam. Unter Mitwirkung von Richard Boschen. .. [es folgen drei weitere Namen] herausgegeben von Julius Haeckel.

1. OW: Haeckel.

z. B.: Materialien für das wirtschaftswissenschaftliche Studium. Herausgegeben von Richard Passow.

1. OW: Passow.

7.3. Ein Sammelwerk, bei dem keine Herausgeber genannt sind, wird im allgemeinen unter dem ersten Verfasser eingeordnet. [Abweichung gegenüber $\S \S 62$ und 65 der PI.]

z. B.: Beiträge zur Thrombosefrage. Von L. Aschoff. . . [es folgen drei weitere Namen.]

1. OW: Aschoff.

7.4. Gesetze, Verordnungen und andere amtliche Veröffentlichungen, in deren Titel kein Urheber, aber Herausgeber, Bearbeiter oder Kommentatoren genannt sind, werden unabhängig von der Bezeichnung und dem Umfang des Kommentars unter dem ersten Herausgeber, Bearbeiter oder Kommentator eingeordnet. [Abweichung gegenüber $\S 217$ der PI.]

z. B.: Das Reichszuwachssteuergesetz. Erläutert von Max Lion.

1. OW: Lion.

\section{Einordnung unter Personennamen}

8.1. Eine Verschmelzung von Präposition und Artikel wird im allgemeinen in den germanischen Sprachen nicht berücksichtigt, in den romanischen dagegen zum Namen gezogen. [Abweichung gegenüber $\S 110$ der PI.]

z. B.: Paul am Ende.

\section{OW: Ende.}

8.2. Bei holländischen Namen, die aus einem Patennamen und einem eigentlichen Familiennamen bestehen, wird der Patenname OW. [Abweichung gegenüber $\S 120$ der PI.]

z. B.: S. K. Thoden van Velzen.

\section{OW: Thoden}

8.3. Ist von verschiedenen für denselben Verfasser in Frage kommenden Namen der eine der wirkliche, der andere ein Pseudonym, so wird das Pseudonym OW. Vom wirklichen Namen wird verwiesen, wenn er ermittelt ist. [Abweichung von $\S 169$ der PI.]

z. B.: Fritz Anders. [Als wirklicher Name ist ermittelt: Max Allihn.]

1. OW: Anders. - Vw von: Allihn.

8.4. Schwankt bei Vornamen deutscher Autoren die Schreibung, so wird jeweils unter der vorliegenden
Form eingeordnet. [Abweichung gegenüber $\S 102$ der PI.]

$$
\begin{aligned}
& \text { z. B.: Mayer, Carl } \\
& \text { Mayer, Conrad } \\
& \text { Mayer, Curt } \\
& \text { Mayer, Karl } \\
& \text { Mayer, Konrad } \\
& \text { Mayer, Kurt }
\end{aligned}
$$

\section{Einordnung unter Sachtiteln}

9.1. Steht am Anfang eines Titels in gewöhnlicher Form eine Präposition oder ein Verschmelzung von Präposition und Artikel, so wird die Präposition oder die Verschmelzung erstes $O W$. Das Substantivum regens wird zweites OW. [Abweichung gegenüber $\S 187$ der PI.]

z. B.: Aus Aachens Vorzeit.

$$
\text { 1. OW: Aus. - 2. OW: Vorzeit. }
$$

z. B.: Zum Gedächtnis an Paul Ewald.

$$
\text { 1. OW: Zum. - 2. OW: Gedächtnis }
$$

9.2. Steht am Anfang eines Titels in gewöhnlicher Form ein Eigenname im Genitiv, so wird er in dieser Form erstes $O W$. Das Substantivum regens wird zweites OW, es sei denn, daß ihm noch weitere Eigennamen im Genitiv vorangehen. Im letzten Fall wird der zweite Eigenname zweites OW. [Abweichung gegenuiber $§ 187$ der PI.]

z. B.: Berlins nähere Umgebung

1. OW: Berlins. - 2. OW: Umgebung.

z. B.: Schaffsteins blaue Bändchen.

1. OW: Schaffsteins. -2 . OW: Bändchen

z. B.: Deutschlands, Österreich-Ungarns und der Schweiz Gelehrte.

1. OW: Deutschlands. -2 . OW: Österreich-Ungarns.

9.3. Von zwei appositionell nebeneinander stehenden, nicht ein Kompositum bildenden Substantiven wird im allgemeinen das erste erstes OW. Das zweite Substantiv wird zweites OW. [Abweichung gegenüber' $\$ 193$ der PI.]

z. B.: Sammlung Göschen.

1. OW: Sammlung. - 2. OW: Göschen.

z. B.: Hundert Blatt künstlerische Zeichenvorlagen.

1. OW: Blatt. - 2. OW: Zeichenvorlagen.

Wo die buchhändlerischen Regeln im Laufe der langen Berichtszeit selbst Veränderungen und Schwankungen unterworfen waren, ist diejenige Regelauslegung gewählt worden, die in der Mehrheit der zugrunde liegenden Alphabete vorzufinden ist. So wird beispielsweise bei einem Titel in gewöhnlicher Form, an dessen Anfang eine Präposition oder eine Verschmelzung von Präposition und Artikel steht, seit dem zweiten Drittel des 19. Jahrhunderts in allen buchhändlerischen Basisbibliographien die Präposition bzw. Verschmelzung erstes Ordnungswort. (Vgl. oben, Abweichung 9.1.) Der Titel „Am häuslichen Herd“ ist also unter „Am" als erstem Ordnungswort $\mathrm{zu}$ finden. In einigen wenigen Alphabeten von Kayser und Heinsius stehen aber solche Titel unter dem Substantiv, beim genannten Beispiel also unter „Herd“. Wegen der sukzessiven Bearbeitung des „GV 1700-1910“ nach dem Alphabet lassen sich solche 
Unstimmigkeiten bereits beim ersten Ordnungswort nur durch Siehe-auch-Hinweise bereinigen, die entweder bereits in den Basisverzeichnissen vorhanden sind, oder von der Redaktion neu geschrieben werden. Beim genannten Beispiel geht den in der Hauptsache unter "Am" verzeichneten Titeln also folgender Siehe-auch-Hinweis voran: „Am s.a. unter dem folgenden Substantiv“.

Überhaupt werden beim Zusammenordnen der verschiedenen Alphabete viele zusätzliche Venweisungen von der Redaktion geschrieben, um die Abweichungen der buchhändlerischen Regeln von den Preußischen Instruktionen auszugleichen. Schon vorhandene Verweisungen werden selbst dann stehengelassen, wenn sie an derselben Stelle des Alphabets auf eine Hauptaufnahme desselben Titels aus einer anderen Quelle treffen. Solche Siehe-Verweisungen werden durch Hinzufügen eines ,,a." (= auch) in Siehe-auch-Hinweise umgeändert, weil nicht auszuschliessen ist, daß sich an der Stelle des Alphabets, auf die verwiesen wird, eine andere Ausgabe derselben Schrift findet.

Häufig sind sogar zwei Haupteintragungen für denselben Titel, also Doppeleintragungen, an der gleichen Stelle im Alphabet des „GV 1700-1910" zu finden: Sie sind bewußt stehen geblieben, wenn es sich zwar offensichtlich um dieselbe Ausgabe eines Werkes handelt, die Titelaufnahmen aus verschiedenen Quellen jedoch unterschiedliche Angaben bezüglich des Erscheinungsjahres, -ortes, Verlages, Herausgebers oder ähnlicher Daten enthalten.

Auch sonst ist bei der Ordnung der Titel möglichst nach dem Grundsatz verfahren worden, alle in den Quellenbibliographien vorhandenen verschiedenen formalen Suchmöglichkeiten zu erhalten und nicht um der Vereinheitlichung willen zu reduzieren. Das bedeutet, daß die Schöne Literatur, die zwischen 1700 und 1821 von Heinsius in den Anhängen für Romane und Schauspiele grundsätzlich, also auch bei bekanntem Verfasser, unter dem Sachtitel eingeordnet worden ist, auch im Alphabet des „GV 1700-1910“ unter ihrem Sachtitel eingeordnet wird. Dadurch, daß Kayser zwischen 1750 und 1832 einen großen Teil derselben Titel in seinen Anhängen bei bekannten Verfassern unter diesen eingeordnet hat, sind diese Titel im Alphabet des „GV 1700$1910^{*}$ ein zweites Mal, nämlich unter ihren Verfassern zu finden.

Die Titel des „Deutschen Anonymen-Lexikons“ von Michael Holzmann und Hanns Bohatta sowie die der „Geschichts- und Romanenlitteratur der Deutschen“" sind in der Regel nur unter ihren Sachtiteln zu finden. Hauptaufnahmen unter oder Verweisungen von ermittel- ten Verfassern sind also nicht neu geschrieben, aber selbstverständlich übernommen worden, wenn sie bereits in anderen Quellen vorgelegen haben, was nicht selten der Fall gewesen ist.

Auch Karten und Pläne sind häufig an zwei Stellen des Alphabets des „GV 1700-1910“" mit Hauptaufnahmen verzeichnet: Unter dem Kartenverfasser bzw. Planfertiger und in vielen Fällen zusätzlich unter einem geographischen Begriff. Letzteres kommt dadurch zustande, daß z. B. im ,Catalog über die im Königlich Bayerischen Haupt-Conservatorium der Armee befindlichen Landkarten und Pläne" und zwischen 1887 und 1910 auch in den Anhängen zu Kaysers Bücher-Lexikon Karten und Pläne alphabetisch-topographisch geordnet worden sind. Dabei wird beispielsweise der Kartentitel „H. Kiepert: Politische Übersichtskarte von Afrika" unter dem geographischen Schlagwort „Afrika“ eingeordnet und im Alphabet des „GV 1700-1910“ unter demselben Schlagwort belassen. Trifft solch ein geographischer Begriff als Schlagwort auf ein gleichlautendes sachliches OW und/oder einen gleichlautenden Verfassernamen, so hat das sachliche OW den Vorrang vor dem Schlagwort und dieses vor dem Verfassernamen.

\section{z. B.: Afrika im Maßstab 1:8000000. [Sachliches OW] \\ Afrika in Wort und Bild. \\ Afrika [Schlagwort] \\ - Handtke, F., General-Karte von Afrika. \\ - Karte, Neue, von Afrika. \\ - Kiepert, H., Politische Übersichtskarte von Afrika \\ Afrika, Emst [Verfasser]}

Unter dem Verfasser Kiepert ist dieselbe Karte dann z. B. mit einer weiteren Hauptaufnahme etwa aus Heinsius oder einer anderen Quelle vertreten.

Abweichungen gegenuber den PI, die zweite und weitere Ordnungswörter betreffen, sind, abgesehen von jenen in den oben genannten Sonderfällen 9.1. bis 9.3., im allgemeinen instruktionsgemäß berichtigt worden. Die falsche Hervorhebung von Ordnungswörtern ist durch Unterstreichen der richtigen Ordnungswörter ungültig gemacht worden; z. B.: Blätter, Rigaer akademische.

Soweit im Zuge der fortschreitenden Ordnungsarbeiten weitere, hier nicht angesprochene Probleme auftauchen, wird die Redaktion durch Schreiben zusätzlicher Verweisungen und Siehe-auch-Hinweise versuchen, den $\mathrm{Zu}$ sammenhang zwischen nicht berücksichtigten und wirklichen Eintragungsstellen herzustellen, soweit dies dem Alphabet nach dann noch möglich ist.

Hans Popst 